\title{
Las lenguas para fines específicos en la Escuela de Comercio de Cádiz hasta 1952
}

\author{
M. Carmen Lario de Oñate \\ Catedrática Escuela Universitaria, Colaboradora Honoraria \\ carmen.lario@uca.es \\ ORCID: https://orcid.org/0000-0003-4974-8004
}

\begin{abstract}
RESUMEN: Actualmente la globalización se presenta como una de las causas de la importancia de los idiomas para desarrollar las profesiones relacionadas con los negocios. Este trabajo muestra que ya desde finales del siglo XIX había interés por formar personas con conocimientos de idiomas y más concretamente de lenguas especializadas en el área del comercio. Ello se evidencia en los programas de las asignaturas de los diferentes planes de estudio de Comercio, los cuales se custodian en el Archivo de la Universidad de Cádiz.

Palabras clave: Escuela de Comercio de Cádiz, Lenguas para fines específicos, Lenguas especializadas, Lenguas aplicadas.
\end{abstract}

\section{Specialized languages at Cádiz Business School until 1952}

ABSTRACT: Globalization is nowadays presented as one of the reasons for the importance of languages to develop business related professions. This study illustrates that since late $18^{\text {th }}$ century there was interest in training people with knowledge of languages and more specifically specialized languages in the area of trade. This fact is evident in the syllabuses of the subjects in the different curricula of Commerce studies, which are preserved in the Archivo de la Universidad de Cádiz.

Keywords: Escuela de Comercio de Cádiz, Languages for specific purposes, Specialized languages, Applied languages.

\section{INTRODUCCIÓN}

El 12 de mayo de 1717 fue la fecha de publicación de la Real Cédula decretada por Felipe V que señalaba a Cádiz como punto de partida y de llegada del tráfico comercial con las colonias americanas, a través del establecimiento de la Casa de Contratación y el Consulado de Mercaderes. A partir de entonces, y hasta el año 1778, el puerto gaditano ostentó el monopolio mercantil indiano que con anterioridad había disfrutado Sevilla. Este hecho hizo de la ciudad un lugar próspero con grandes oportunidades debido fundamentalmente al ingente movimiento de barcos que arribaban desde las colonias americanas y al creciente establecimiento de casas 
de comercio que negociaban con esos productos que llegaban de tierras lejanas, entre otros factores. La ciudad vivió entonces una etapa de enorme relevancia en el mundo de los negocios. Cádiz era una ciudad cosmopolita que acogía una población variopinta procedente no sólo de otras ciudades españolas sino también de diferentes países europeos que entendieron la oportunidad de negocio que este lugar ofrecía. La actividad comercial era un pilar fundamental en la economía de la ciudad; la importación, exportación y reexportación dependía de comerciantes, dependientes, tratantes, y de muchas profesiones que tenían una estrecha relación con el mundo de los negocios.

La importancia mercantil de la ciudad queda patente en un pequeño apunte que aparece en la carta que Alexander Munro, Cónsul General de Inglaterra en España, envía desde Cádiz a Ralph Woodford en $1785^{1}$ : «De lo poco que he visto esta es la ciudad más capital de España, fundamentalmente por su vasto comercio, el cual se desarrolla de la manera más extraordinaria». Con estas líneas Munro nos presenta una ciudad cuyo motor principal es el mundo del comercio. Juan F. Peyron (1962, p. 799), de nacionalidad francesa, también hizo una referencia a Cádiz en este sentido: «...no se puede calcular el número de comerciantes ricos y poderosos que lo habitan, o, por mejor decir, toda la ciudad es comerciante». El Barón de Bourgoing (1790, p. 69) destacó también la actividad mercantil que esta ciudad generaba. Los habitantes de Cádiz se dedican casi exclusivamente al comercio; esto es evidente simplemente observando el número de embarcaciones en el puerto, que generalmente cuenta con unas seiscientas o setecientas, aunque cuando yo estuve en la ciudad no pasaban de trescientas.

La ciudad, por tanto, necesitaba personas con unos sólidos conocimientos sobre el intercambio de mercancías, su comercialización y venta.

\section{ESTUDIOS DE COMERCIO}

Según Antón Solé (1959) durante el siglo XVIII se impartían ciertas materias vinculadas al comercio en el Colegio de la Compañía de Jesús, establecida en Cádiz desde el siglo XVI (Antón Solé 1959). La expulsión de los jesuitas en 1767 fue probablemente el detonante para que el 5 de enero de 1771 se creara una comisión de diputados que visitara al Gobernador con el fin de tratar sobre la creación del establecimiento de cátedras de comercio (Guillén Tato 1941), aunque esta petición no prosperó. Posteriormente, en 1787, se recibe en los Consulados del Mar una Real Orden en la que se explicitan «las disposiciones acordadas por S.M. el Rey para crear la enseñanza mercantil y cuyo plan debía ser elevado al superior conocimiento por los respectivos consulados» (García Gutiérrez 1898, p. 69).

Ya en 1803 y siguiendo instrucciones de la Real Orden del 11 de mayo de ese año se crea en Cádiz una comisión para planificar la creación de una Escuela Mercantil. El 26 de septiembre de ese año se convocaron dos cátedras de matemáticas, que se cubrieron en mayo del siguiente año, momento en el que se nombraron asimismo dos profesores de lenguas vivas: uno de lengua francesa y toscana, y otro de lengua inglesa (García Gutiérrez 1898).

${ }^{1}$ The National Archives (Kew, Londres). FO 72/29, f. 98.

DOI del artículo:

https://doi.org/10.25267/Cuad investig fondos arch UCA.2020.i2.06 
Precisamente, unos meses después, en 1804, Salvador Joseph Solari, Traductor del Real Tribunal del Consulado, pronunció en dicha institución un discurso en el que expuso la necesidad de la creación de una Academia Mercantil (Solari 1804).

Figura 1. Portada del Discurso sobre el comercio presentado por Salvador José Solari, 1804

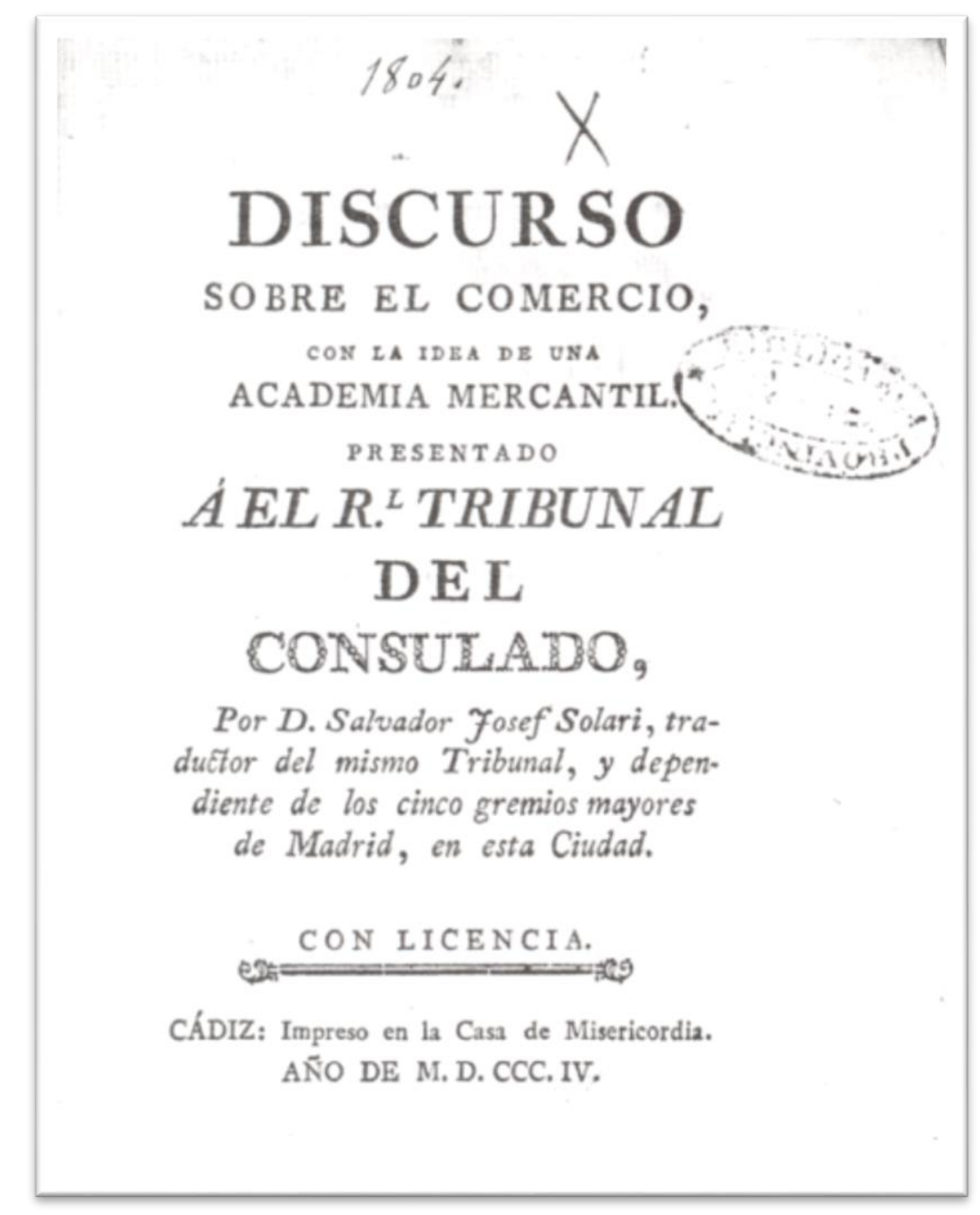

Por fin, el 2 de enero de 1819 se inauguran oficialmente los estudios de Comercio en Cádiz (Guilloto 1994). Desde entonces y hasta la actualidad estos estudios han pasado por distintas ubicaciones en la ciudad, diferentes centros de enseñanza y, desde luego, diversos planes de estudio.

La situación privilegiada de Cádiz como punto intermedio en el sistema de intercambios de mercancías entre Europa y América propició que se convirtiera en un lugar muy atractivo para el aprendizaje comercial de una forma práctica en una casa de comercio. El parentesco solía ser una forma muy usual de llegar a formar parte de una compañía en la que instruirse; hijos y sobrinos se iniciaban en los temas comerciales en los negocios de la familia. Muchos se desplazaban desde sus lugares de origen; ese es el caso de Juan Cólogan, que llegó a Cádiz procedente de Dublín entre 1720 y 1724 y también el del escocés Carlos Gordon, que llegó en 1789 a la casa de su tío Diego Duff para instruirse en el comercio, entre otros (Lario 2000). 
Pero ¿cuáles eran esos conocimientos tan necesarios para un comerciante de aquella época? Wyndham Beawes, cónsul británico en Cádiz, publicó en 1745 en Dublín la magistral obra Lex Mercatoria Rediviva en la que ofrecía amplia información sobre el comercio para los hombres de negocios. En uno de los apartados expone detenidamente qué variedad de temas necesitaba dominar un comerciante del siglo XVIII. Se partía de lo más elemental como saber leer y escribir correctamente o utilizar con dominio las reglas de la aritmética hasta llegar a otros conocimientos más especializados como el uso de los documentos mercantiles pertinentes, saber llevar la contabilidad en los diferentes libros, dominar todo lo concerniente a los fletes y los seguros, etc. Entre todos los conocimientos necesarios Beawes incluía el dominar idiomas, tanto en su uso común y cotidiano como en un uso más específico en todas las áreas antes mencionadas. Este apunte nos parece de suma importancia ya que el texto tiene, como ya se ha mencionado arriba, más de dos siglos y medio.

En 1850 un Real Decreto estableció de manera oficial la enseñanza del comercio en España. En Cádiz esta formación se impartía en la Escuela Oficial Elemental de Industria, Comercio y de Náutica. El plan de estudios era el siguiente (Lario 1999, p. 47):

* Matemáticas elementales

* Metrología universal y Sistemas monetarios

* Partida doble, Teneduría de libros y Cálculos mercantiles

* Elementos de Economía política, Balanza universal y Seguros y aranceles reformados

* Geografía fabril y mercantil y nociones de derecho comercial

* Lengua Inglesa

* Lengua Francesa

Posteriormente, en 1857, hubo una reforma que reorganizaba los estudios mercantiles que se impartían en España, así se crearon las Escuelas de Comercio que podían ser Elementales o Superiores. El ciclo elemental tenía una duración de tres años y, a su finalización, otorgaba el título de Perito Mercantil; una vez obtenido ese título se podía acceder al segundo ciclo, el superior, de un año, que capacitaba para recibir el título de Profesor Mercantil. En el primer curso se impartían dos idiomas: Lengua Inglesa y Lengua Francesa. En el segundo: segundo curso de Lengua Francesa y primer curso de Lengua Alemana o Italiana. En tercero se impartía el segundo curso de Lengua Inglesa y el segundo de Lengua Alemana o Italiana. Es decir, se recibía formación en tres idiomas, aunque se impartían cuatro. En el ciclo superior no había idioma alguno.

En 1887, tras gestiones de diversas instituciones gaditanas, se concede a Cádiz, junto a otras ciudades, el establecimiento de una Escuela Elemental de Comercio con el siguiente plan de estudios, en el que se vuelven a impartir cuatro idiomas, pudiendo el alumno cursar tres de ellos. 
Figura 2. Plan de estudio de 1887

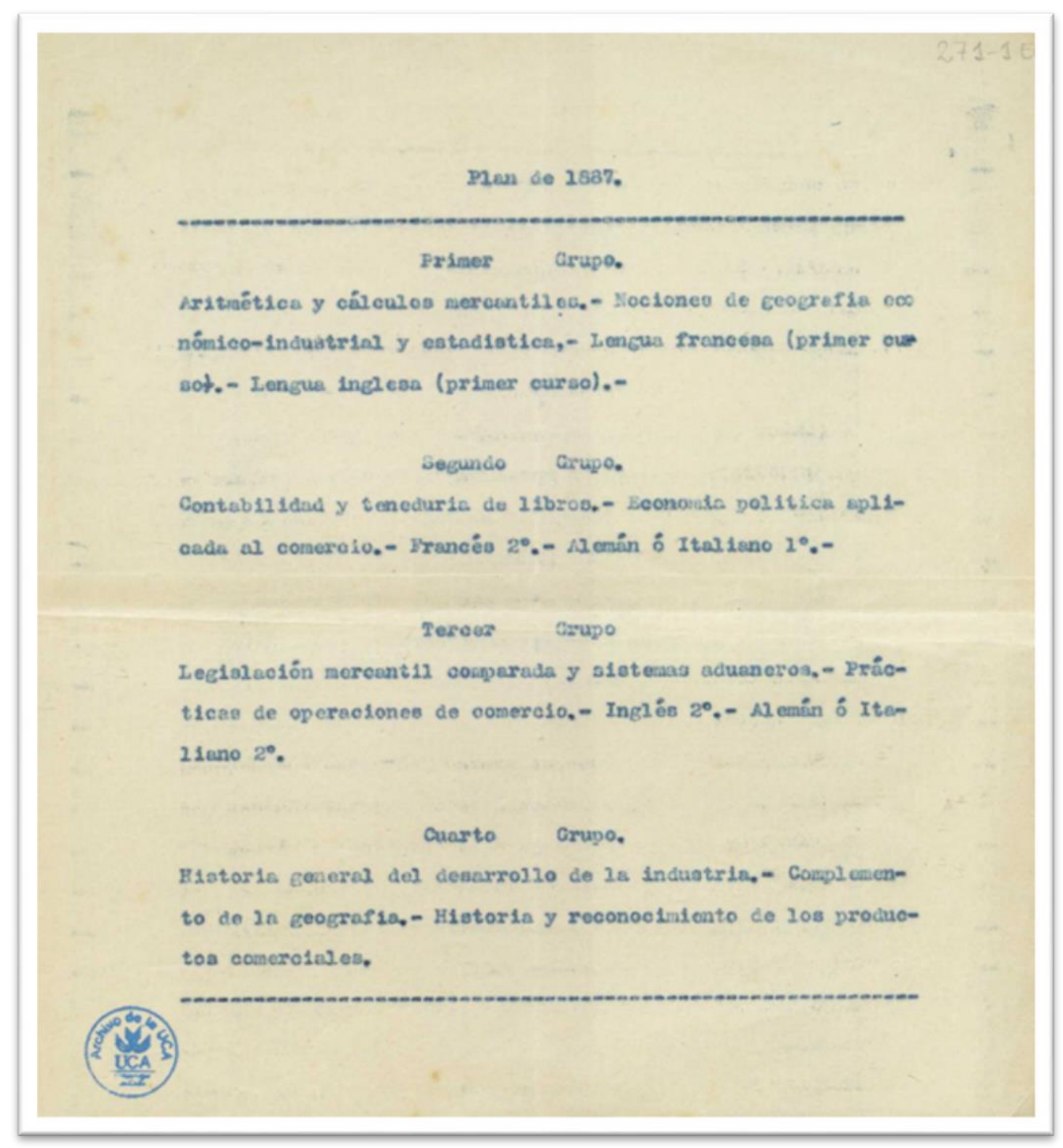

Fuente: Archivo de la Universidad de Cádiz (AUCA), C-271-1 EC

La asignatura de Inglés desde enero de 1893 la impartía el Catedrático Interino Agustín García Gutiérrez. Él era perito mercantil y empleado del Banco de España y ocupó la plaza de Catedrático Interino de Lengua Inglesa en la Escuela de Comercio de Cádiz, plaza que había sido convocada por Real Orden el 10 de enero de 1893, y ganada por él por oposición en 1899 (Guilloto 1994). Este profesor era un gran conocedor de la actividad comercial de Cádiz. En 1895 el Ateneo de Cádiz convocó un certamen científico-literario con distintos premios. El que otorgaba la Cámara de Comercio, Industria y Navegación de Cádiz proponía el siguiente tema: «Medios de fomentar el desarrollo comercial, industrial y marítimo en Cádiz» (prosa). El profesor García Gutiérrez obtuvo el premio con el trabajo presentado bajo el lema «To be or not to be, that is the question» (Lario 2006). 
Figura 3. Nombramiento Catedrático Interino A. García Gutiérrez (1893)

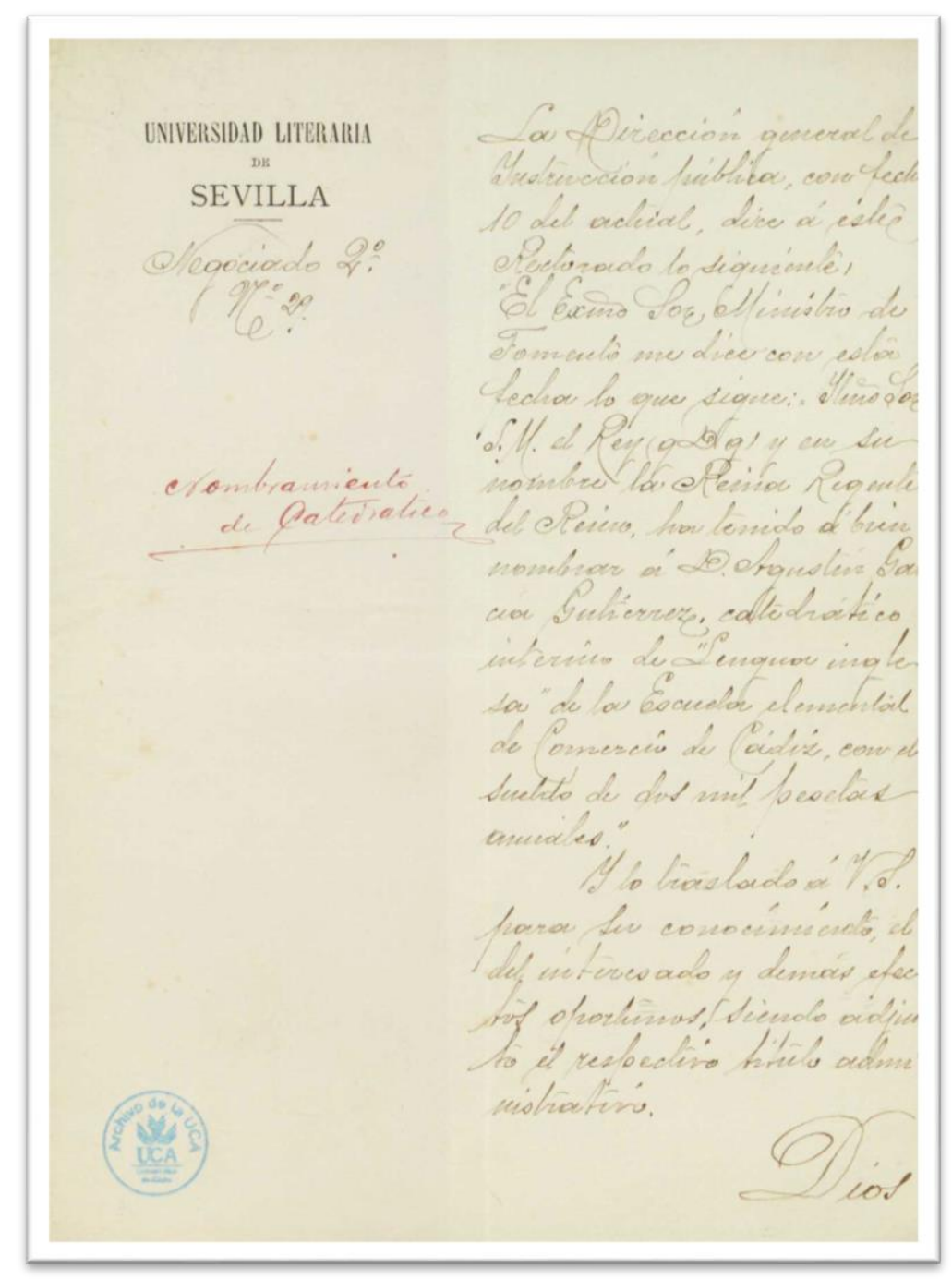

Fuente: Archivo de la Universidad de Cádiz (AUCA), C-268-3 EC

García Gutiérrez (1898, p. 101) expone en la siguiente cita muy claramente su idea relativa a la enseñanza de los idiomas en estos centros:

Dos formas pueden ser adoptadas para la enseñanza de idiomas: el método científico y el teórico práctico. El primero puede ser el indicado para los Institutos y otros centros en los cuales vienen a encajar las escabrosidades de la gramática comparada con la fonética, la morfología y otras derivaciones de la lingüística; pero para la enseñanza comercial, para la industria y el comercio o sea para los alumnos que se han de dedicar a dichos ramos, lo que necesitan saber son conocimientos de idiomas para hablarlo y para escribirlo, es decir la conversación y la correspondencia por métodos que resulten prácticos y sencillos.

Por tanto, ya a finales del siglo XIX se perfilaba la importancia de enseñar idiomas en las Escuelas de Comercio adaptándolos a las futuras necesidades profesionales de las personas dedicadas a las actividades mercantiles, lo que en la actualidad se denomina Lenguas para Fines Específicos. Estas lenguas especializadas tienen como objetivo la enseñanza a 
individuos adultos de una lengua no materna que, con un propósito instrumental, puedan servir como medio para el desarrollo de una actividad profesional.

El programa de Lengua Inglesa de primero, segundo y tercer curso editado por el profesor García Gutiérrez en 1893 recoge los temas que se impartían en cada uno de los cursos. El primero de ellos era de Inglés General, pero los otros dos estaban dedicados a la enseñanza de Inglés para Fines Específicos, concretamente Inglés Comercial. Este mismo profesor publicó asimismo en forma de libro las Contestaciones Sintéticas al Programa de Lengua Inglesa en el año siguiente (García Gutiérrez 1894).

El 8 de julio de 1897 el Director de la Escuela, reunida la Junta, «dirigió expresivas frases de saludo a los nuevos Sres. Catedráticos que venían a formar parte del Claustro con motivo de la elevación a Superior de este centro de Enseñanza ${ }^{2}$, la Escuela Elemental pasaba a ser Escuela Superior de Comercio de Cádiz. Posteriormente, en 1903 vuelve a haber una reforma, pero se siguen impartiendo lenguas extranjeras, en este caso inglés, francés y alemán. Ya entonces las asignaturas de idiomas utilizaban una metodología estructurada en destrezas, impartiéndose en primer lugar destrezas receptivas escritas (lectura y traducción) y ya en el año posterior, productivas escritas (escritura) y orales (conversación).

Figura 4. Plan de estudios 1903

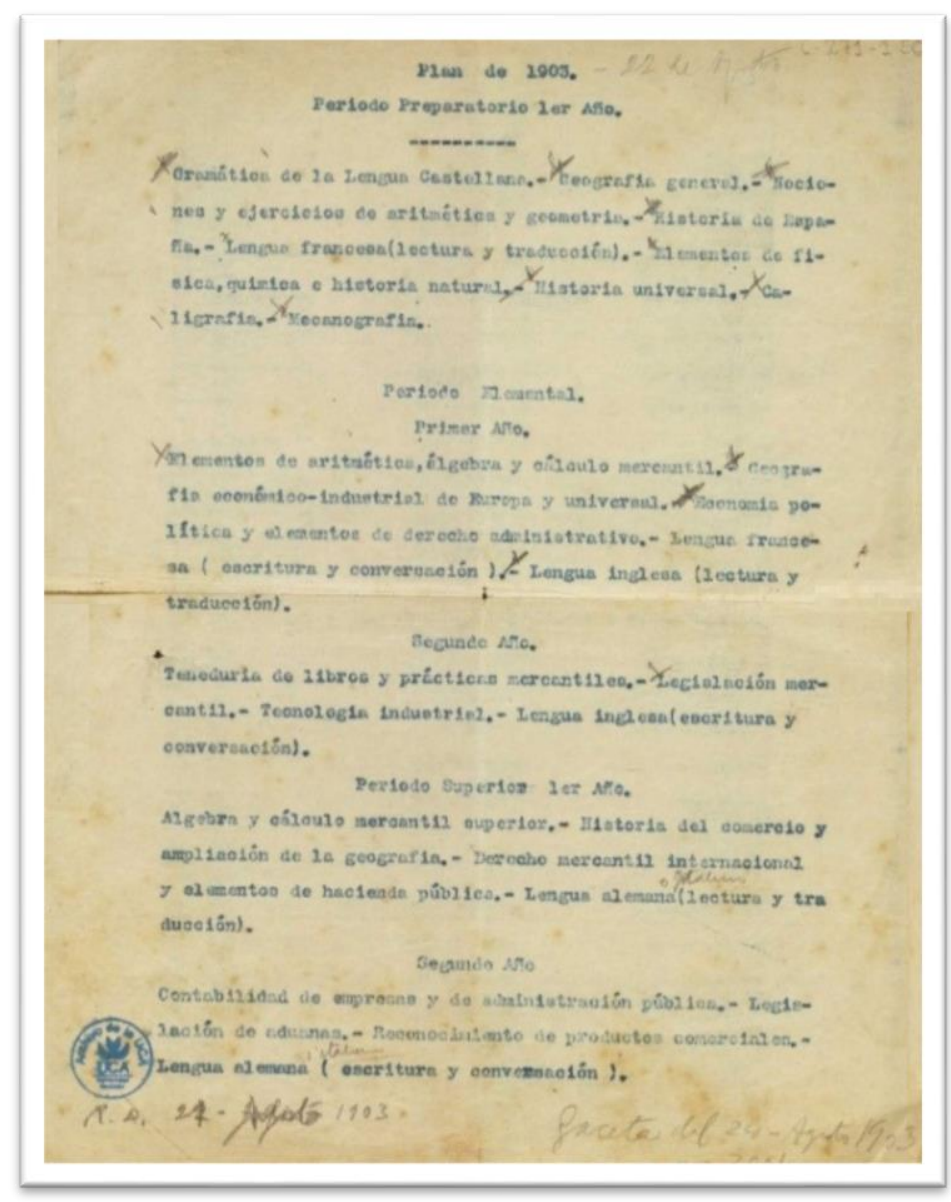

Fuente: Archivo de la Universidad de Cádiz (AUCA), C-271-1 EC

\footnotetext{
${ }^{2}$ Archivo de la Universidad de Cádiz (AUCA), L-101 EC, Libro de Actas, 8 julio 1897. DOI del artículo: 
En 1912, el plan de estudios incluye inglés, francés, alemán y árabe. Ese plan, en principio, no distingue entre las distintas destrezas lingüísticas, simplemente el nivel y el curso, aunque en anotaciones manuales sí se especifican las prácticas de los diferentes idiomas. Asimismo, se detalla que el árabe que se enseña es el vulgar y no el clásico, no hay que olvidar que en ese año se firmó el Protectorado Español de Marruecos.

Figura 5. Plan de estudios 1912

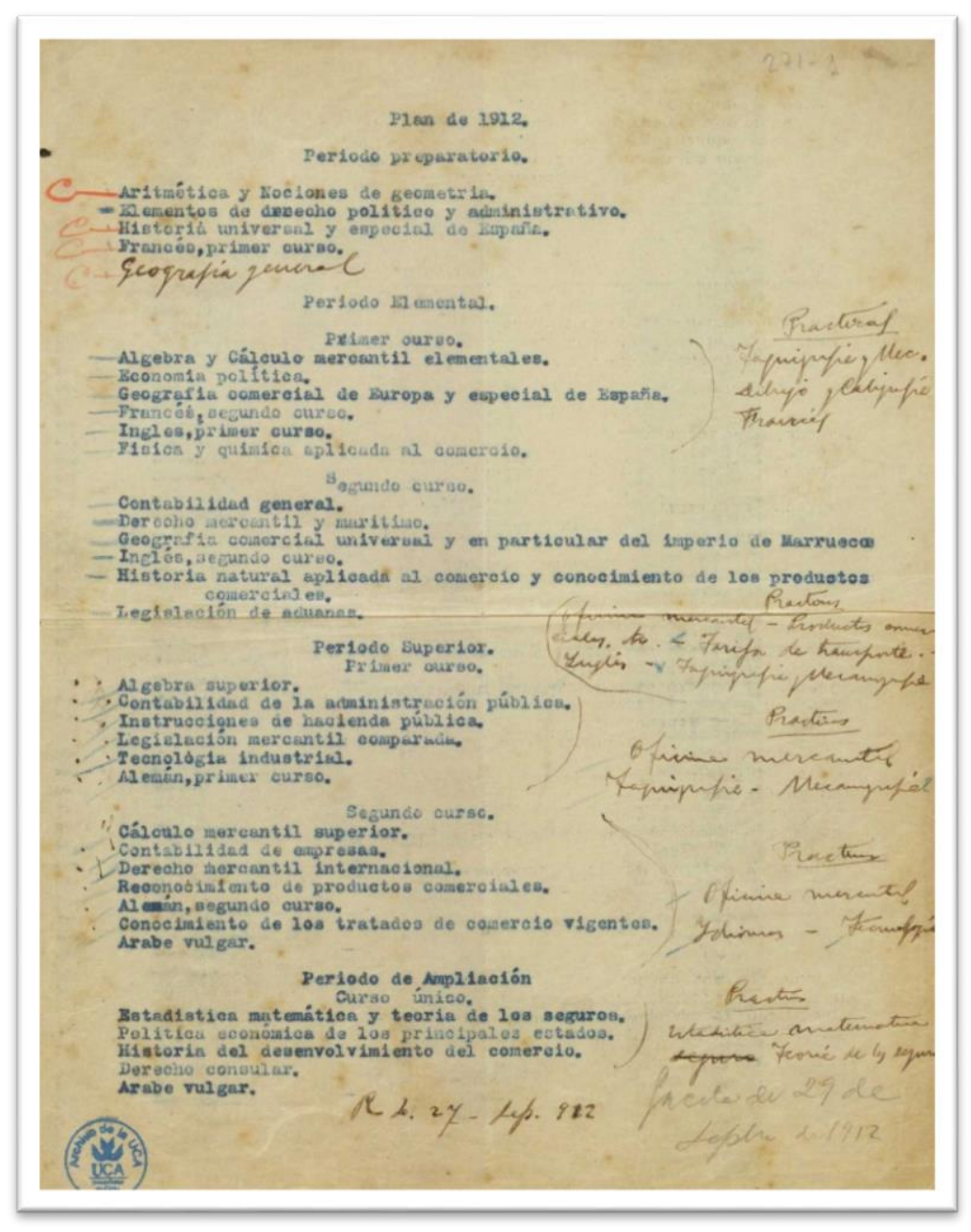

Fuente: Archivo de la Universidad de Cádiz (AUCA), C-271-1 EC

El 16 de abril de 1915 el Real Decreto del Ministerio de Instrucción Pública y Bellas Artes establece que los estudios mercantiles comprenderán tres grados: elemental o de vulgarización, profesional o técnico y superior o de altos estudios. Este último, a su vez, comprende tres especialidades: la Docente para la formación del profesorado de Escuelas de Comercio «para formar al hombre de empresa capaz de concebir, planear, ordenar y dirigir los grandes negocios comerciales»; la especialidad Actuarial; y la de Ingeniería Comercial para formar profesionales que posteriormente ocupen cargos de Directores, Asesores, Secretarios de grandes empresas, instituciones financieras o la Administración Pública.

La especialidad docente consta de tres secciones: Letras, Ciencias e Idiomas. En esta última se imparte alemán e italiano en dos cursos con frecuencia diaria e inglés y francés en 
un curso también diariamente. El artículo 21 de este Real Decreto de 1915 establece que en el primer curso el alumno debe iniciarse en la traducción y la composición para dedicarse en el segundo a la cultura general y la práctica comercial en conversación y correspondencia. Queda, por tanto, establecido oficialmente que la lengua extranjera debe ser aplicada a los fines profesionales concretos con el fin de proporcionar a los estudiantes la competencia comunicativa que les permita desenvolverse en el entorno profesional.

Es indudable la importancia que se da al conocimiento y dominio de las lenguas en la formación de las personas dedicadas al mundo de la empresa, por ello el artículo 55 de dicho Real Decreto de 1915 recomienda «aumentar en todas las Escuelas de Comercio, sucesivamente, la enseñanza de mayor número de lenguas vivas, bien formando parte de los estudios obligatorios o con carácter voluntario». Una de estas lenguas a que se refiere el artículo es el árabe vulgar que se encomienda impartir al profesorado de la asignatura Marruecos y Posesiones Españolas.

Con el fin de completar la formación del estudiante y redactar la memoria que deben presentar para la reválida de Intendente Mercantil, el artículo 67 del mencionado decreto establece la creación de bolsas de viaje para aquellos que deseen realizar dicho trabajo en el extranjero. Estas becas se ganaban por oposición.

Posteriormente, el Boletín Oficial del Ministerio de Instrucción Pública y Bellas Artes publica el 10 de diciembre de 1925 un número extraordinario dedicado a la enseñanza mercantil. En él se desglosan las asignaturas en su vertiente «oral» y prácticas. Los idiomas, inglés, francés y alemán, se imparten en clases «orales» y en las prácticas se especifica que deben estar dedicadas a la correspondencia y documentación comerciales. Es necesario dominar la lengua extranjera en su faceta general y también en la aplicada al conocimiento mercantil.

\section{CONCLUSIÓN}

El interés por el conocimiento de idiomas en la formación de profesionales dedicados al comercio queda reflejado en todos los planes de estudio presentados aquí y pertenecientes a siglos anteriores. Es destacable el número tan alto de idiomas diferentes que se impartían, no solo el inglés y el francés que tan en boga estuvieron posteriormente, sino también el alemán, el italiano e incluso el árabe. Este dato parece reflejar que durante esos años no había una lengua franca omnipotente como ocurre hoy día, sino que las personas dedicadas al comercio debían manejar varios idiomas para poder comunicarse con sus socios extranjeros. Es también destacable la importancia que se otorgó a las distintas destrezas, tanto orales como escritas, y la relevancia de enseñar una lengua aplicada a esos conocimientos que necesita un profesional para desenvolverse en el mundo de los negocios. Todo ello demuestra que, ya desde finales del siglo XIX, la Administración era consciente de las necesidades de comunicación de unos futuros profesionales que, en el desarrollo de su trabajo, requerirían establecer relaciones con otros comerciantes, con mayoristas, consignatarios, empresarios, etc. de otras nacionalidades. Para llevar a cabo esos contactos deberían utilizar un lenguaje tanto oral como escrito, tanto de uso cotidiano formal como informal, general y también específico, una lengua extranjera 
aplicada al comercio. Para materializar todo ello los profesores de lenguas extranjeras de las Escuelas de Comercio pusieron entonces en marcha unos principios didácticos que continúan vigentes hoy día.

\section{REFERENCIAS BIBLIOGRÁFICAS}

BeAwes, W., 1754. Lex Mercatoria Rediviva: or the Merchants Directory. Dublín: J. Williams.

Bourgoing, J. P., 1790. Travels in Spain with a new, accurate and comprehensive View of the present State of the Country. Vol II. Dublín: Fitzpatrick.

GARCÍA GUTIÉRREZ A., 1995. Medios de fomentar el desarrollo comercial, industrial y marítimo de Cádiz. J. M. Barragán y J. Torrejón (Eds.). Cádiz: Ingrasa.

García Gutiérrez, A., 1893. Programa de Lengua Inglesa. Cádiz: Imp. de la Revista Médica.

García Gutiérrez, A., 1894. Contestaciones Sintéticas al Programa de Lengua Inglesa. Cádiz: Imp. de la Vda. de A. Choza.

García Gutiérrez, A., 1894. English and Spanish Commercial-Vocabulary. Cádiz: Imp. de la Revista Médica.

GARCíA GutiÉRreZ, A., 1898. La enseñanza mercantil en España y en el extranjero. Cádiz: Tip. Cabello y Luzón.

Guillén TAto, J. F., 1941. Índice sistemático de acuerdos de las Actas Capitulares. 17171807. Cádiz: Cerón.

Guilloto y GonZÁlez, F., 1994. La carrera de comercio en Cádiz. Cádiz: Servicio de Publicaciones de la Universidad de Cádiz.

LARIO de OÑATE, M. C., 1999. El Inglés para Fines Específicos en España a finales del siglo XIX y principios del XX. A. Bocanegra Valle, M. C. Lario de Oñate, y P. López Zurita (eds.). Enfoques Teóricos y Prácticos de las Lenguas Aplicadas a las Ciencias y a las Tecnologías. Cádiz: Dpto. Filología Francesa e Inglesa y Tesitex, pp. 46-51.

LARIO de OÑATE, M. C., 2000. La colonia británica e irlandesa en Cádiz a finales del siglo XVIII. Cádiz: Servicio Publicaciones Universidad de Cádiz.

LARIO de OÑate, M. C., 2006. Agustín García Gutiérrez: Un precursor en la enseñanza de idiomas. Actas del IX Congreso de la Sociedad Española de Historia de las Ciencias y de las Técnicas. Cádiz: SEHCYT, pp. 873-879 (tomo II).

Peyron, J. F., Nuevo viaje a España 1772-1773. En: J. García Mercadal, 1962, Viajes de extranjeros por España y Portugal, v. III. Madrid: Aguilar.

SOlARI, S. J., 1804. Discurso sobre el comercio, con la idea de una Academia Mercantil, Cádiz: Imprenta Casa de la Misericordia. 